\title{
An Improved Images Watermarking Scheme Using FABEMD Decomposition and DCT
}

\author{
Noura Aherrahrou and Hamid Tairi \\ University Sidi Mohamed Ben Abdellah, \\ Faculty of Sciences, Dhar El mahraz, \\ LIIAN, Department of Informatics, \\ Fez, Morocco \\ noura.ah@hotmail.fr, htairi@yahoo.fr
}

\begin{abstract}
In this paper, we propose a new robust digital image watermarking scheme which integrates the Discrete Cosine Transform (DCT) and the Fast and Adaptive Bidimensional Empirical Mode Decomposition (FABEMD). The use of the FABEMD decomposition is motivated by the fact that FABEMD has better quality than any other decomposition technique in extracting intrinsic components known as Bidimensional Intrinsic Mode Functions (BIMFs) and residue. In the proposed approach, the watermark is embedded in the DCT coefficients of the residue, in order to achieve better performance in terms of perceptually invisibility and the robustness of the watermark. Experimental results and comparison analysis demonstrate that our method has better performance than the traditional watermarking method operating in the DCT domain.
\end{abstract}

Keywords: DCT, FABEMD, BIMFs, Watermarking.

\section{Introduction}

The fast development of the Internet in recent years has made it possible to easily create, copy, transmit, and distribute digital data. Consequently, this has led to a strong demand for reliable and secure copyright protection techniques for digital data. Digital watermarking has been proposed as valid solution for this problem.

In order to be successful, the watermark should be invisible and robust to premeditate any spontaneous modification of the image. It is very important to be robust against common image processing operations such as filtering, additive noise, resizing, cropping, and common image compression techniques.

Watermarking techniques can be categorized in different ways. They can be classified based on 1) the type of watermark being used (the watermark can be a visually recognizable logo or a sequence of random numbers) or 2) domain where the watermark is applied (spacial domain or the frequency-domain). 
The most popular approaches for the image watermarking are the frequencydomain approaches. In these schemes, the image is being transformed via some common frequency transform and watermarking is achieved by altering the transform coefficients of the image. The transforms that are usually used are the DCT, DFT and the DWT. A question that raises in such approaches is the number and the position of the altered coefficients in the frequency representation of the image. Many different ideas have been presented, most of them originating from Cox's et al. system [5].

The method proposed by Cox's et al. computes the N x N DCT coefficients for an $\mathrm{N} x \mathrm{~N}$ image. The watermark of length $\mathrm{n}$ is placed into the $\mathrm{n}$ highest magnitude coefficients of the transform matrix. The motivation for choosing the higher value coefficients is that they represent the low frequency regions of an image, which contain most of the perceptually significant image information. Also, the human visual system attaches more resolution to the low frequency spectral components. Furthermore, it has been observed that common signal processing operations and distortions affect the perceptually insignificant regions of an image, which correspond to high-frequency components. So, the watermark has to be inserted in the low-frequency components.

Further performance improvements in the Cox's proposed method may be achieved by using the FABEMD decomposition. The use of FABEMD in Cox's method and the embedding of the watermark in the DCT coefficients of the residue is supported by the fact that FABEMD has better quality than any other decomposition technique in extracting intrinsic components (BIMFs) which contain the different frequency parts of the image from high to low frequency (from BIMFs to residue).

\section{FABEMD (Fast and Adaptive Bidimensional Empirical Mode Decomposition)}

\subsection{FABEMD Overview}

Empirical Mode Decomposition (EMD) is first developed by Huang et al. [1] and has shown to be a powerful tool for decomposing nonlinear and nonstationary signals. The concept of EMD is to decompose the signal into a set of zero mean functions called Intrinsic Mode Functions (IMF) and a residue. This decomposition technique has also been extended to analyze two-dimensional (2D) data/images, which is known as bidimensional EMD (BEMD). In EMD or BEMD, extraction of each IMF or BIMF requires several iterations. Hence, the extreme detection and interpolation at each iteration make the process complicated and time consuming. The situation is more difficult for the case of BEMD, which requires 2D scattered data interpolation at each iteration. For some images it may take hours or days for decomposition.

To overcome these limitations of BEMD, a novel approach called Fast and Adaptive BEMD (FABEMD) was proposed recently by Bhuiyan et al. [2, 3]. It substitutes the 2D scattered data interpolation step of BEMD by a direct envelop estimation method and limits the number of iterations per BIMF to one. In this technique, spatial 
domain sliding order-statistics filters, namely, MAX and MIN filters, are utilized to obtain the running maxima and running minima of the data. Application of smoothing operation to the running maxima and minima results in the desired upper and lower envelopes respectively. The size of the order-statistics filters is derived from the available information of maxima and minima maps.

\subsection{FABEMD Algorithm}

Figure 1 illustrates the different steps of the FABEMD. Let the original image be denoted as I, a BIMF as BIMF $F_{i}$, and the residue as R. In the decomposition process ith BIMF BIMF $F_{i}$ is obtained from its source image $S_{i}$, where $S_{i}$ is a residue image obtained as $\mathrm{S}_{\mathrm{i}}=\mathrm{S}_{\mathrm{i}-1}-\mathrm{BIMF}_{\mathrm{i}-1}$ and $\mathrm{S}_{1}=\mathrm{I}$. It requires one or more iterations to obtain $\mathrm{BIMF}_{\mathrm{i}}$, where the intermediate temporary state of BIMF in jth iteration can be denoted as $\mathrm{F}_{\mathrm{Tj}}$. With the definition of the variables, the steps of the FABEMD process can be summarized as follows:

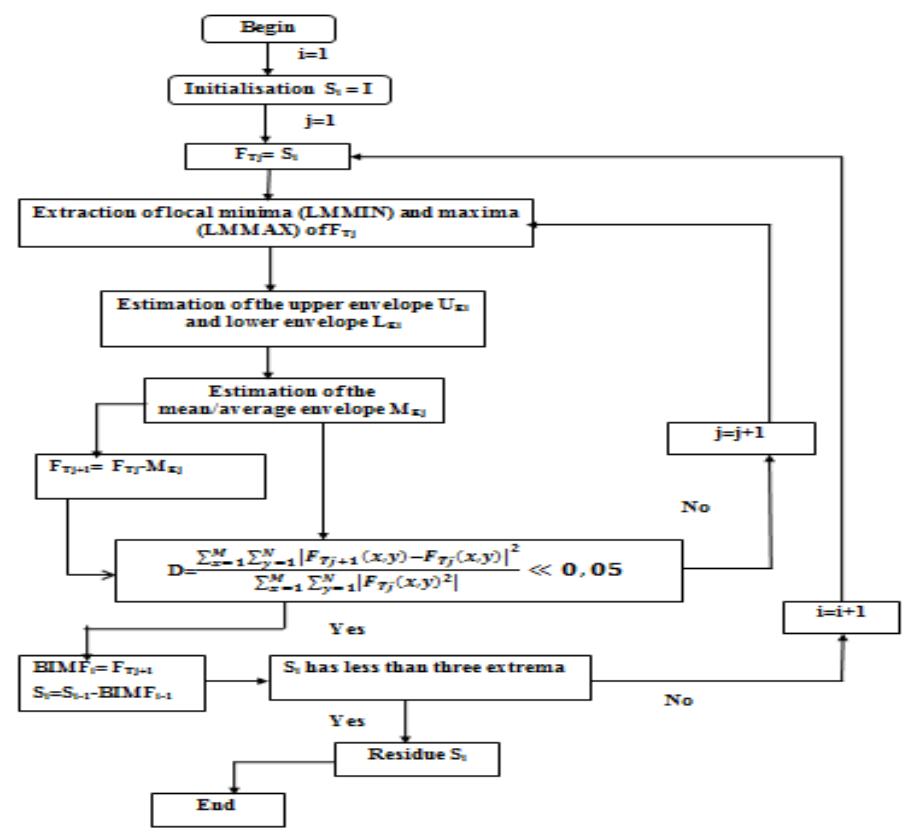

Fig. 1. FABEMD algorithm

Figure 2 shows an example of FABEMD decomposition. 


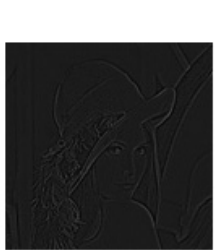

BIMF 1

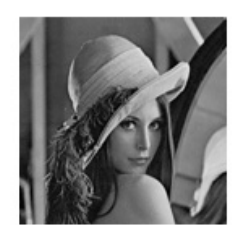

$256 \times 256$ - pixel Lena image

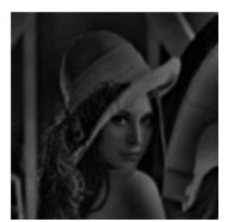

BIMF 2

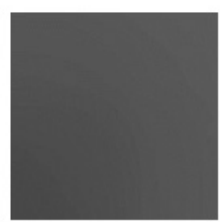

Residue

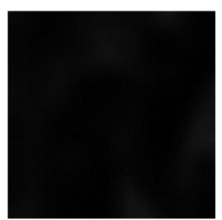

BIMF 3

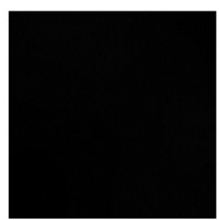

BIMF 4

Fig. 2. Result of the FABEMD decomposition of Lena image

\section{Proposed Approach}

\subsection{Watermark Embedding}

The watermark $X=\left\{x_{1}, x_{2}, \ldots x_{n}\right\}$ consists of a pseudo-random sequence of $M$ length.

To insert the watermark in the host image, the first step is to decompose the image into BIMFs and Residue, then calculated DCT, followed by introduced the watermark on the selected coefficients of the Residue.

The watermark will be introduced from the $\mathrm{L}+1$ coefficient to the $\mathrm{M}+\mathrm{L}$ coefficient of the DCT coefficients range.

These coefficients generate the vector $\mathrm{T}=\left\{\mathrm{t}_{\mathrm{L}+1} \ldots \ldots \ldots \mathrm{t}_{\mathrm{L}+\mathrm{M}}\right\}$ and will be modified according to:

$$
\mathrm{t}_{\mathrm{L}+\mathrm{i}}^{\prime}=\mathrm{t}_{\mathrm{L}+\mathrm{i}}+\alpha\left|\mathrm{t}_{\mathrm{L}+\mathrm{i}}\right| \mathrm{x}_{\mathrm{i}} .
$$

where $\alpha$ is the watermark strength, which determine the invisibility of the watermark.

These coefficients will be the elements of the vector $T^{\prime}=\left\{t_{L+1}^{\prime}, t_{L+2}^{\prime} \ldots \ldots t_{L+M}^{\prime}\right\}$ the modified coefficients.

Finally, the vector $\mathrm{T}^{\prime}$ is reinserted into the original DCT coefficients according with the original order, and with the IDCT it is obtained the watermarked Residue which will be added to BIMFs to produce the watermarked host image. 
Therefore, the watermark embedding steps are represented in figure 3 followed by a detailed explanation.

1. Decompose image into BIMFs and residue.

2. Generate the watermark $\mathrm{X}$ which has to be inserted.

3. Calculate the DCT of the Residue.

4. Generate the coefficient vector $\mathrm{T}$.

5. Modify the coefficient vector $\mathrm{T}$ according to the $\mathrm{X}$ values to obtain $\mathrm{T}$ '.

6. Swap the original coefficients $(\mathrm{T})$ by the modified in the vector $\mathrm{T}$ '.

7. Calculate the IDCT of the Residue to obtain the watermarked residue.

8. Build the watermarked image by adding all BIMFs and the watermarked residue

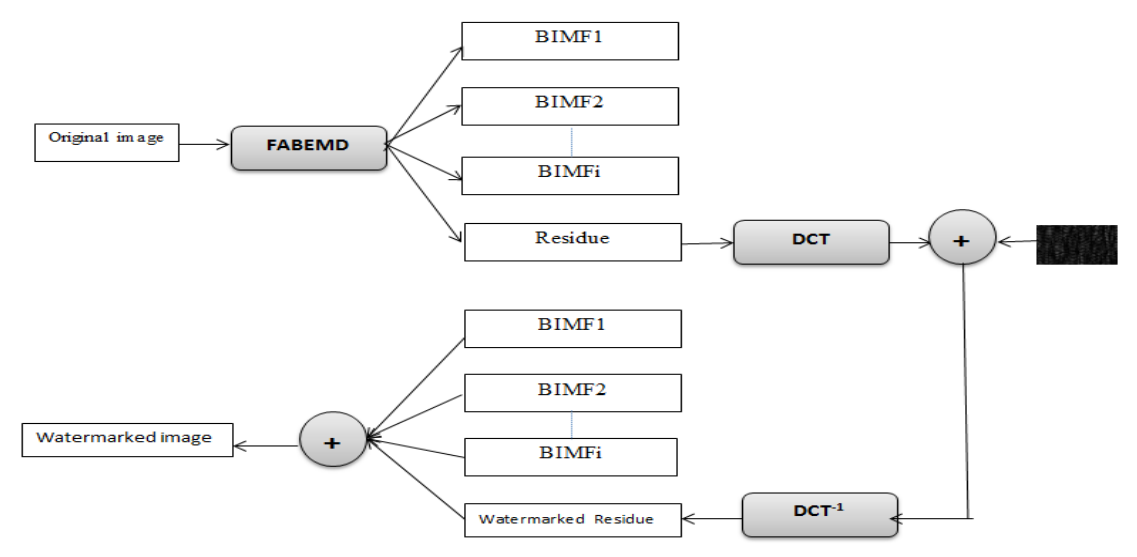

Fig. 3. Watermark embedding scheme

\subsection{Watermark Detection}

The first step to detect the watermark of a given image I* consists of calculating the DCT transform. The DCT coefficients of $\mathrm{I}^{*}$ from $\mathrm{L}+1$ to $\mathrm{L}+\mathrm{M}$ are selected to form the vector of marked coefficients and perhaps modified $\mathrm{T}^{*}=\left\{\mathrm{t}_{\mathrm{L}+1}^{*}, \mathrm{t}_{\mathrm{L}+2}^{*}, \ldots \ldots \mathrm{t}_{\mathrm{L}+\mathrm{M}}^{*}\right\}$

To detect the mark it's necessary to correlate the marked coefficients and perhaps modified $\mathrm{T}^{*}$ with the watermark Y. The correlation formula to be used is defined by:

$$
\mathrm{z}=\frac{\mathrm{YT}^{*}}{\mathrm{M}}=\frac{1}{\mathrm{M}} \sum_{\mathrm{i}=1}^{\mathrm{M}} \mathrm{y}_{\mathrm{i}} \mathrm{t}_{\mathrm{L}+\mathrm{i}}^{*}
$$

Where yi is the watermark to be verified and $t_{\mathrm{L}+\mathrm{i}}^{*}$ are the coefficients of the marked DCT and perhaps modified. 
The DCT coefficients which are inside the vector $\mathrm{T}$ are always the coefficients which were obtained ignoring the first $\mathrm{L}$ elements and taking the next $\mathrm{M}$ elements, then the previous formula will be simplified as:

$$
\mathrm{z}=\frac{\mathrm{YT}^{*}}{\mathrm{M}}=\frac{1}{\mathrm{M}} \sum_{\mathrm{i}=1}^{\mathrm{M}} \mathrm{y}_{\mathrm{i}} \mathrm{t}_{\mathrm{i}}^{*}
$$

The correlation $\mathrm{z}$ can be used to determine whether a given mark is present or not, $\mathrm{z}$ is simply compared to a predefined threshold $\mathrm{Tz}[5]$, whereas $\mathrm{z}$ is computed for each of the watermarks and that with the largest correlation is assumed to be the one really present in the image.

To detect the watermark into a given image the following steps are shown in figure 4 .

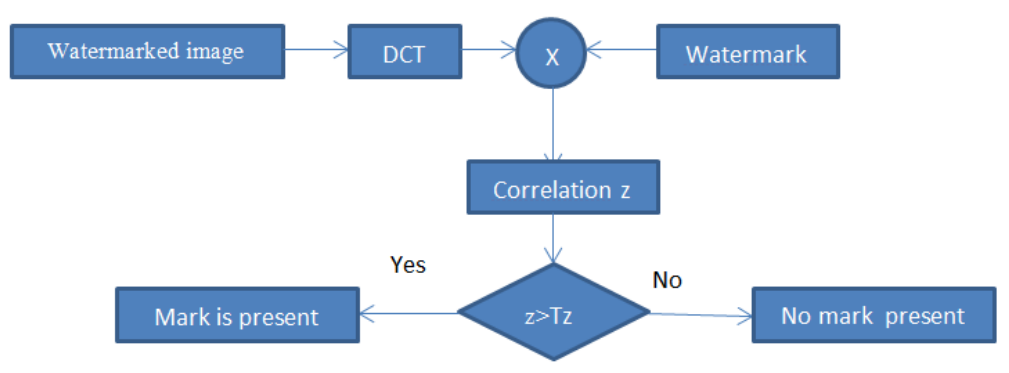

Fig. 4. Watermark detection scheme

\section{Experimental Results}

In this section the results of our study are shown. Several experiments are done to evaluate the effectiveness of the presented watermarking algorithm.

\subsection{Invisibility of the Watermark}

In this section the invisibility of the watermark is evaluated.

The PSNR is popularly used to measure the similarity between the original image and the watermarked image. While higher PSNR usually implies higher fidelity of the watermarked image.

As can be seen in table1, for traditional method, we observe that if we fix the watermarking parameters $\mathrm{L}$ and $\mathrm{M}$, then smaller watermarking strength $\alpha$ Results in higher robustness of watermark process, while the unreasonably big watermarking strength $\alpha$ Results in the watermark perceptually visible in the watermarked image. Here the invisibility of the watermark is demonstrated to show the successful use of the FABEMD in the traditional scheme. 


\subsection{Robustness of the Method against Attacks}

To check if the proposed scheme is robust it has been implemented some attack functions to compare the expected results with the obtained results.

The mark will be specified by $\alpha=0.2$ and $L=16000, M=16000$. Furthermore, to check the detection process behavior will be used a group of 100 watermarks, only one will be the correct (Only watermark number 40 is correct) while the others will be randomly generated.

Table 1. Comparison between our method and Cox's method

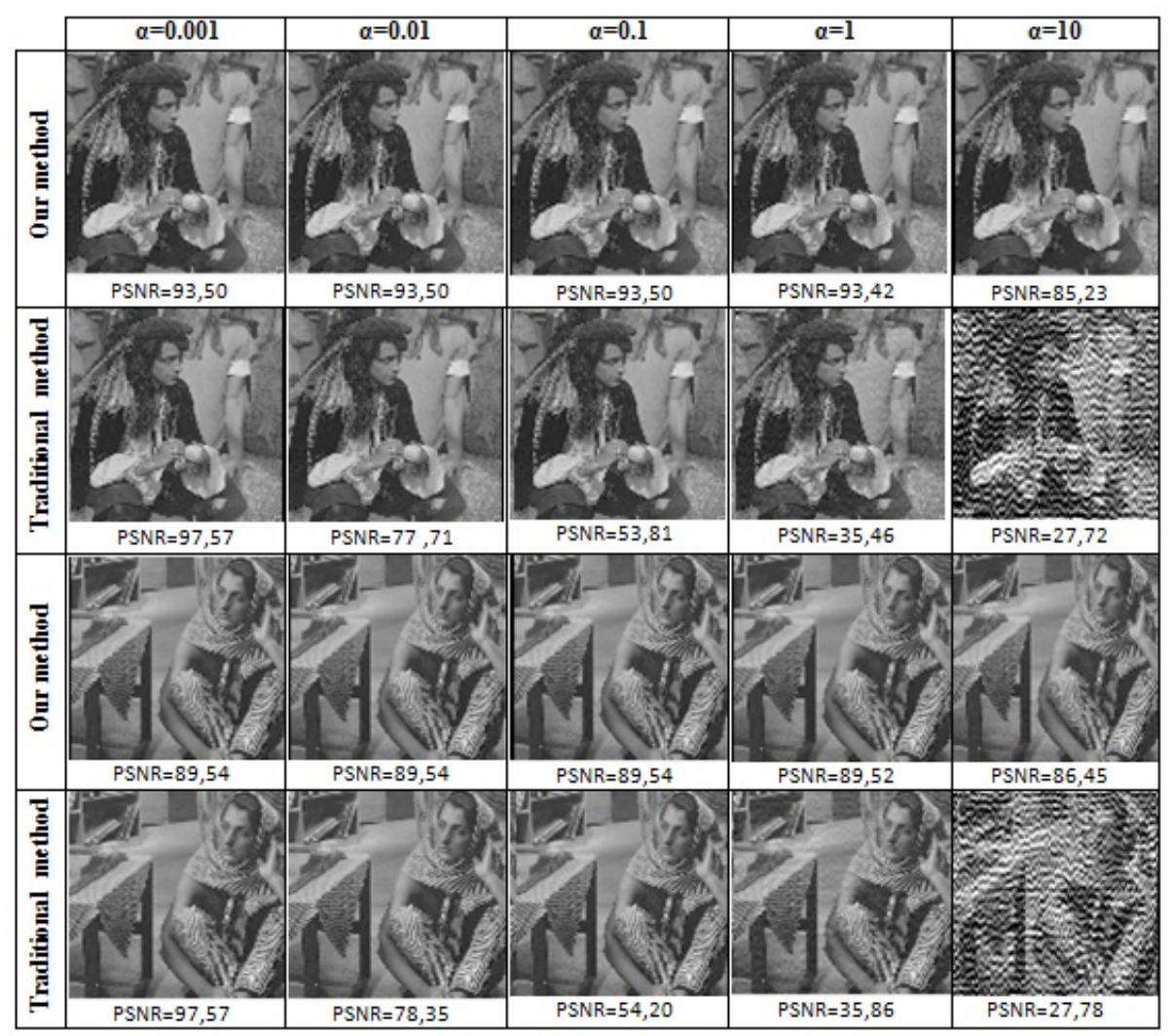




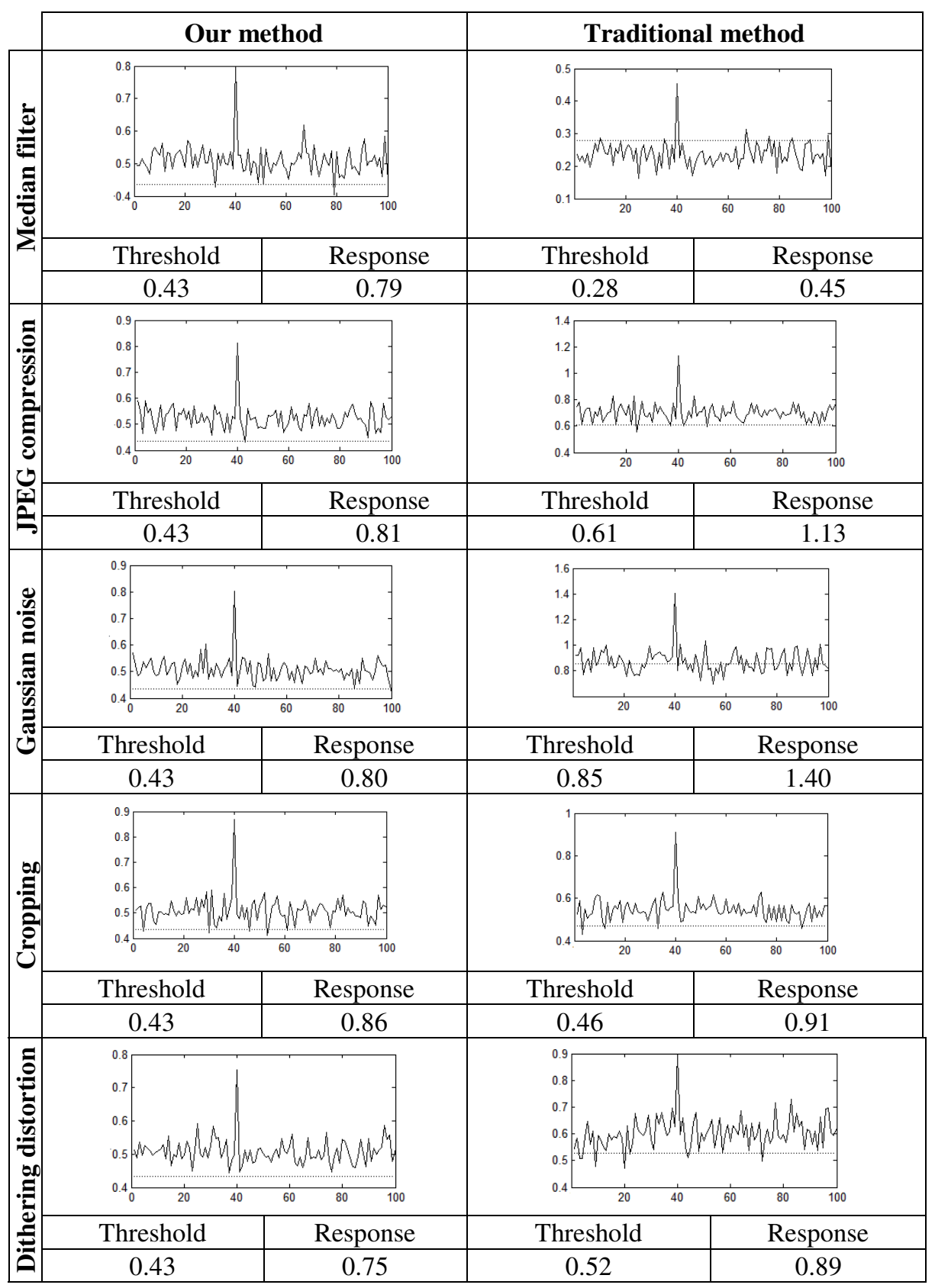

Fig. 5. Detector responses on 100 randomly generated watermarks, after $512 X 512$ pixel Lena watermarked image been attacked with gaussian noise, dithering distortion, cropping, JPEG compression and median filter using Cox's method and the proposed method. Only watermark number 40 is correct 


\section{Conclusion}

We have presented a new robust digital image watermarking scheme based on joint FABEMD-DCT. Our scheme is shown to be resistant against several signal processing techniques, including dithering distortion, median filtering, Gaussian noise, cropping, and JPEG compression. Furthermore, we show that the implementation of the FABEMD algorithm leads to better performance in terms of invisibility of the watermark compared to traditional method.

\section{References}

1. Huang, N.E., Shen, Z., Long, S.R., et al.: The empirical mode decomposition and the Hilbert spectrum for nonlinear and non-stationary time series analysis. Proceedings of the Royal Society A 454(1971), 903-995 (1998)

2. Bhuiyan, S.M.A., Adhami, R.R., Khan, J.F.: Fast and adaptive bidimensional empirical mode decomposition using order-statistics filter based envelope estimation. EURASIP J. Adv. Signal Process 2008, 1-18 (2008)

3. Bhuiyan, S.M.A., Adhami, R.R., Khan, J.F.: A novel approach of fast and adaptive bidimensional empirical mode decomposition. In: Proc. IEEE Int. Conf. on Acoustics, Speech and Signal Processing (ICASSP), Las Vegas, USA, March 31-April 4, pp. 1313-1316 (2008)

4. Cox, I., Kilian, J., Thompson Leighton, F., Shamoon, T.: Secure Spread Spectrum Watermarking for Multimedia. IEEE Trans. on Image Processing 6(12) (December 1997)

5. Barni, M., Bartolini, F., Cappellini, V., Piva, A.: A DCT Domain System for Robust Image Watermarking. Signal Processing 66, 357-372 (1998) 